\title{
Statistical Properties of Inter-Series Mixing in Helium: From Integrability to Chaos
}

\author{
R. Püttner ${ }^{1}$, B. Grémaud ${ }^{2}$, D. Delande ${ }^{2}$, M. Domke ${ }^{1}$, M. Martins $^{1}$, A. S. Schlachter ${ }^{3}$, and G. Kaindl ${ }^{1}$ \\ ${ }^{1}$ Institut für Experimentalphysik, Freie Universität Berlin, Arnimallee 14, D-14195 Berlin-Dahlem, Germany \\ ${ }^{2}$ Laboratoire Kastler-Brossel T12 E1, Université Pierre et Marie Curie, 4 Place Jussieu, 75005 Paris, France \\ ${ }^{3}$ Lawrence Berkeley National Laboratory, 1 Cyclotron Road, Berkeley, CA 94720, USA
}

(February 8, 2001)

\begin{abstract}
The photoionization spectrum of helium shows considerable complexity close to the doubleionization threshold. By analyzing the results from both our recent experiments and ab initio threeand one-dimensional calculations, we show that the statistical properties of the spacings between neighboring energy levels clearly display a transition towards quantum chaos.
\end{abstract}

Since the work of Poincaré, it is known that the general classical three-body problem has only global constants of motion, such as energy and angular momentum. It is thus non-integrable, since there are not enough nontrivial constants of motion to allow an analytical solution. This typically implies that the phase space is a mixture of regular and chaotic dynamics. Celestial mechanics abounds with examples, e.g. the prototypical earthmoon-sun system [1]. The dynamics of three charged particles is superficially similar since the force law scales also as $1 / r^{2}$, but with two possible signs of the coupling constant. The actual dynamics of the two electrons in helium - the simplest three-body quantum system - is largely chaotic, even for the simplified situation with the nucleus fixed in space. Nonetheless, at low energies the quantum states of helium occur in seemingly regular progressions, labeled by sets of approximately good quantum numbers, and even the doubly excited states have largely been classified [2]. What are then the manifestations of the underlying classical chaos in the quantum spectrum of helium? This is a fundamental question in quantumclassical correspondence, with regard to the nature of semi-classical approximations in the presence of chaos, and in quantum chaos itself. What will be the signatures of the onset of quantum chaos? One expects that the approximate quantum numbers, overviewed e.g. in Ref. [2], will cease to function, as series of states overlap and mix so strongly that there are essentially no good quantum numbers left, except for the ordering of states by their energies. The doubly excited states of helium are resonances, which will overlap and interact strongly when chaos sets in, giving rise to Ericson fluctuations wellknown in phenomenological nuclear theory [3]. It is the purpose of this Letter to present new results from experiment and theoretical modeling, which clearly show that the threshold to this new regime has now been passed for the first time in a three-body quantum system with known Hamiltonian.

The ${ }^{1} \mathrm{P}^{o}$ doubly excited states of helium can be described in Herrick's classification scheme by $N, K_{n}$, with $N(n)$ denoting the principal quantum number of the in- ner (outer) electron, and $K$ the angular-correlation quantum number [4]. For fixed $N$, the various $n, K$ series converge to the single-ionization threshold $I_{N}=-4 / N^{2}$ (in Rydberg units). Starting with $N=5$, the lowest states of the series lie below $I_{N-1}$. As a consequence, they act as perturbers of the $N-1$ series, leading to interferences [5], which can be reproduced by numerically complicated $a b$ initio calculations [6]. While up to the $N=8$ threshold, $I_{8}$, the effects of the perturbers are quite simple, from $I_{9}$ on, the increasing proliferation of perturbers tends to complicate the spectra increasingly, and Herrick's classification starts to break down, at least for a large fraction of states [7].

The most intense series in the spectrum are the principal series with $K=N-2$. Since $K \cong-N<\cos \Theta>$, where $\Theta$ is the angle from the nucleus to the two electrons, $\Theta$ approaches $\pi$ for principal series with large $\mathrm{N}$. Therefore, the experimentally observed series can be related in the semiclassical limit - based on Gutzwiller's trace formula [8] - to periodic orbits of the collinear $e Z e$ configuration, with both electrons on opposite sides of the nucleus. It is well known that the classical dynamics of the $e Z e$ configuration is strongly chaotic in radial, but stable in angular direction. One can thus expect a mixing of series with different $N$ but constant $N-K$, i.e. constant number of bending quanta with respect to a collinear $e Z e$ configuration [2]. In other words, for highly excited series, $N-K$ is expected to be approximately a good quantum number, while states with the same $N-K$, but different $(N, n)$ strongly interact [6].

There are numerous semiclassical studies of helium based on Gutzwiller's trace formula (see e.g. Ref. [2]), which aim at understanding the structure of quantum dynamics in terms of its classical counterpart. The present work focuses on the random-matrix approach [9], which deals with universal aspects of quantum chaos, i.e. the general features present in all chaotic quantum systems. We compare the present experimental spectra close to $I_{9}$ with the results of our calculations and find excellent agreement. In particular, we show that the statistics of nearest-neighbor level spacings can be well reproduced 
by a simple random-matrix model adapted to intermittency [10], even though $N-K$ is still a good quantum number. This model mixes regular and chaotic spectra and corresponds to an interaction between regular Rydberg series and chaotic perturbers. Using a simplified one-dimensional (1D) model of helium, we reproduce the transition from the regular to a fully chaotic regime.

The experiments were performed at beamline 9.0.1 of the Advanced Light Source (ALS) in Berkeley, California, using photons with a spectral resolution of $\cong 2 \mathrm{meV}$ (FWHM) and a setup described in Ref. [11]. The calculations were performed with the complex-rotation method on a Cray C98, with details given in Ref. [6].

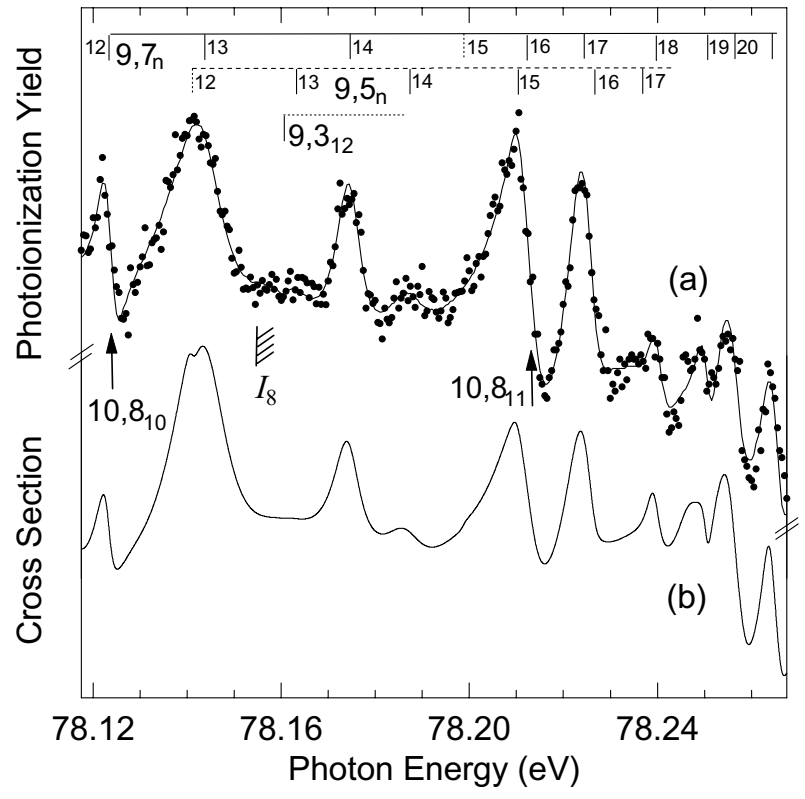

FIG. 1. (a): Double-excitation spectrum of He in the region of the $9,7_{n}$ principal Rydberg series, with perturbers $10,8_{10}$ and $10,8_{11}$ (vertical arrows). The solid line through the data points represents the best fit. Assignments of the resonances are made by vertical-bar diagrams on top, including resonances of the secondary series $9,5_{n}$ and $9,3_{n}$. (b): $A b$ initio calculated spectrum in the same region.

Figure 1(a) shows the spectrum of the ${ }^{1} \mathrm{P}^{o}$ double excitations in helium in the energy region just below $I_{9}$ from 78.1175 to $78.2675 \mathrm{eV}$, with considerably improved resolution and signal-to-noise ratio as compared to previous results [12]. In Fig. 1(b), we also show the theoretical spectrum, convoluted with a Gaussian of $2-\mathrm{meV}$ width. In the least-squares fit of the measured spectrum, the theoretical values for linewidth and Fano $q$-parameter were used, but the energy positions and intensities of the lines were adjusted to allow for possible deviations between experiment and theory, spectral drifts, and non-linearities. Details of this analysis have been given elsewhere [11]. As a result, the calculated spectrum matches the experimental data very well. We note that some resonances of the $9,7_{n}$ principal and the $9,5_{n}$ secondary series re- veal Fano parameters $|q| \gg 1$ (up to $|q| \cong 7$, with negative sign, for $\left.9,7_{14}\right)$, very different from the values found for the principal and secondary series below the $I_{5}$ to $I_{8}$ thresholds, with $|q| \leq 1$ [12]. However, even these unexpected $q$-values are described well by our calculations. This makes us confident that the energy levels obtained numerically are sufficiently accurate to perform a statistical analysis on the nearest-neighbor spacings (NNS), as discussed in the following.

The NNS distribution, $P(s)$, measures the distribution of energy spacings between consecutive eigenstates. In order to allow a comparison of large energy spacings far away from threshold with small energy spacings close to threshold, the spectra were unfolded, i.e. the energy spacings were divided by an energy-dependent mean level spacing [13], so that the mean unfolded spacing, $s$, is unity. For a single unperturbed Rydberg series (or, more generally, for any regularly spaced energy levels), this would lead to a constant unfolded level spacing $s=1$, i.e. to $P(s)=\delta(s-1)$, where $\delta$ is the delta-function. When a good quantum number exists in a system, the spectrum can be divided into various non interacting, but overlapping series. The nearest neighbor of a given state belongs then typically to another series, and the energies of neighboring states are thus completely uncorrelated, giving rise to a Poisson distribution, $P(s)=\exp (-s)$. This happens e.g. in integrable multi-dimensional systems, but also if several irregular series overlap without interaction. For a fully chaotic system, the prediction for $P(s)$ can be derived from random-matrix theory. Due to time reversal symmetry of the system, a Gaussian Orthogonal Ensemble (GOE) of random matrices [13] is used resulting in $P(s)$ to be very close to a Wigner distribution, $P(s)=\frac{\pi}{2} s \exp \left(-\pi s^{2} / 4\right)$. Since the number of energy levels for the statistical analysis is rather limited in the present case, one obtains a rather noisy $P(s)$. We therefore use the cumulative NNS distribution, $N(s)=\int_{0}^{s} P(x) d x$, leading to $N(s)=1-\exp (-s)$ and $N(s)=1-\exp \left(-\pi s^{2} / 4\right)$ for a Poisson and a Wigner distribution, respectively.

The spectra were analyzed by two different procedures: (i) globally by considering all resonances regardless of the series to which they belong; (ii) individually for each series associated with a given value of $N-K$.

We first analyze by the global procedure (i) the calculated levels in the energy region 78.1000-78.2662 eV, where there are 112 resonances, most of them from the $N=9$ series, with perturbers from higher series. The cumulative NNS distribution is shown in Fig. 2(a) together with a cumulative Poisson distribution. The agreement is very good, showing that an approximately good quantum number exists. This is not surprising, since one can identify experimentally states with different $N-K$ (see Fig. 1(a)). Occasionally, these states are mixed with other series (in the vicinity of perturbers), but $N-K$ is still approximately a good quantum number. This is also partly 
true for other series not observed in the experiment [14]: the series with positive $K$ are almost independent, while those with negative $K$ are significantly coupled. In the full spectrum, the various $N-K$ series are superimposed with rather weak mixing, resulting mainly in an uncorrelated ensemble of levels, which thus obeys Poisson statistics. This complies with the stability of the $e Z e$ collinear configurations with respect to off-collinear perturbations.

Hence, a relevant data analysis must be done individually for each $N-K$ series (procedure (ii)). The cumulative NNS distributions, $N(s)$, obtained in this way, are shown in Fig. 2(b) for resonances below $I_{4}$ and in Fig. 2(c) for those below $I_{9}$. The statistical accuracies are limited due to the relatively small number of data points with $71(60)$ spacings for $I_{4}\left(I_{9}\right)$. Moreover, for $I_{9}$, only series with $K$ between 0 and 8 were unfolded because of $K$-mixing for negative $K$ values, while for $I_{4}$ all series are used. The $I_{4}$ distribution clearly reflects the quasiregularity in this energy region, as it is very close to a step function, which results from integrating over a deltafunction. This is the statistical analogue to the fact that the spectrum below $I_{4}$ is composed only of $N=4$ states and can be described by single-channel quantum defect theory. Below $I_{9}$, the situation has slightly changed, although the distribution still does not match a cumulative Wigner distribution. It means that the relative density of chaotic perturbers with $N>9$ has increased as well as their interaction with the various Rydberg series. The bold line in Fig. 2(c) shows $N(s)$ using only the experimentally observed series $N-K=2$ and 4 . Due to the small number of 17 spacings, the statistics are relatively poor, but it is striking that the bold line closely follows the solid line. As a consequence, the spectrum in Fig. 1(a) represents the first experimental verification of a transition of the NNS distribution towards quantum chaos in a three-body Coulomb system.

The complex numerical calculations for 3D helium render it difficult to obtain enough spacings for a quantitative analysis in case of $N>9$. However, the fact that $N-K$ remains approximately a good quantum number means that the bending motion can be essentially frozen in the $e Z e$ configuration. In other words, the quantum properties are essentially those of $1 \mathrm{D}$ helium, a system that has only two degrees of freedom. This leads to much simpler numerics allowing higher ionization thresholds to be reached. We have therefore calculated the resonances of $1 \mathrm{D}$ helium below $I_{9}, I_{13}$, and $I_{17}$ using a new approach (banded sparse matrix representation of the Hamiltonian in a $1 \mathrm{D}$ perimetric basis, in the spirit of Ref. [6]) that represents a significant improvement over previous methods [15].

In order to improve statistics, we calculated spacings in a given energy region for slightly different values of the nuclear charge $Z$, from $1 / Z=0.45$ to $1 / Z=0.55$, in steps of 0.01 . These values are statistically uncorrelated and sufficiently close to $Z=2$ of helium, so that the av- erage density of states and the classical dynamics do not change significantly. Figs. 2(d)-(f) show the cumulative NNS distributions for states below $I_{9}, I_{13}$, and $I_{17}$, respectively, as well as the cumulative Wigner distribution and the $3 \mathrm{D}$ result for $I_{9}$. The results demonstrate that the statistical level properties are essentially the same for $1 D$ and $3 D$ helium and they illustrate the transition from an irregular regime $\left(I_{9}\right)$, with a distribution intermediate between a step function and a cumulative Wigner distribution, to a chaotic regime $\left(I_{17}\right)$, with a distribution that is almost Wigner-like. For $I_{17}$, the lack of large spacings is the only remnant of regularity.

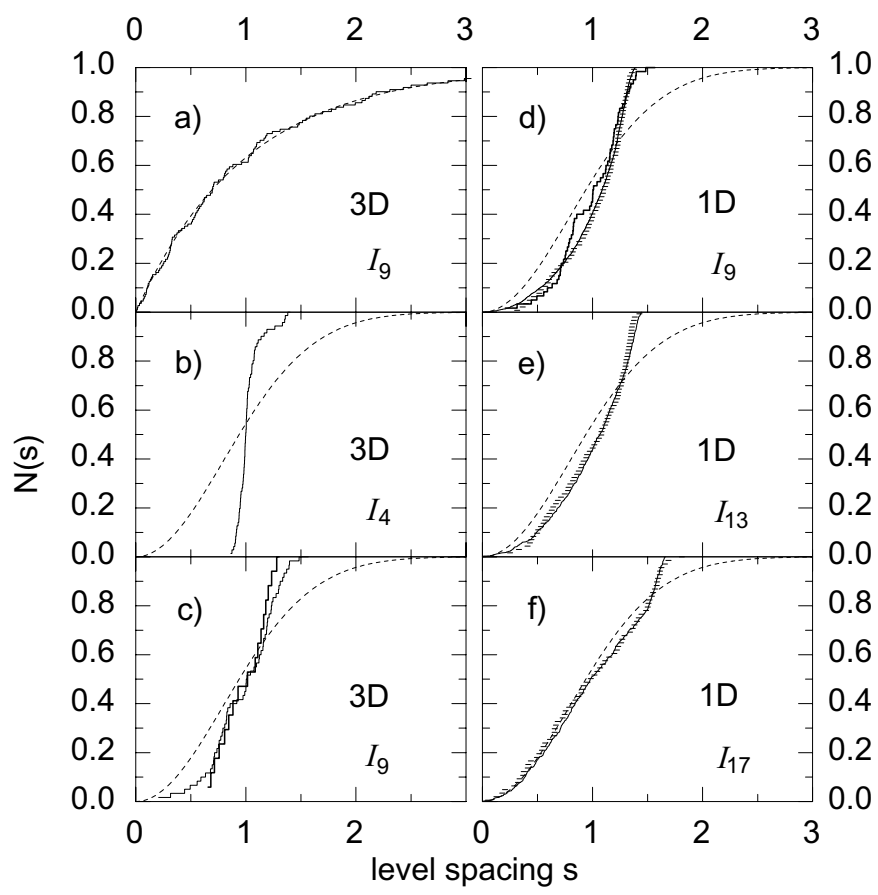

FIG. 2. (a)-(c): Cumulative NNS distributions for the ${ }^{1} \mathrm{P}^{\mathrm{o}}$ states of helium below (b) $I_{4}$ and (a,c) $I_{9}$. (a): Global analysis using all levels below $I_{9}$. The data (solid line) agree very well with a cumulative Poisson prediction (dashed line). (b) and (c): Distributions below $I_{4}$ and $I_{9}$, respectively, obtained by analyzing separately individual series with different $K$. For $I_{9}$ (c), the bold line is the distribution derived from experiment. (d)-(f): Cumulative NNS distributions for singlet states of 1D helium (horizontal bars) below $I_{9}, I_{13}$, and $I_{17}$, respectively. The solid lines are the fit results (see text). The bold solid line in (d) is the NNS distribution for states of 3D helium below $I_{9}$. The dashed lines in (b)-(f) represent the cumulative Wigner distribution.

This behavior can be understood in a quantitative way by the model of Zakrzewski et al. [10], which was developed to understand the NNS statistics of the hydrogen atom in a magnetic field, whose spectrum is quite similar to that of helium in the sense that 'chaotic' perturber states interact with a regular series. In this model, the Hilbert space is composed of two subspaces, a 'regular' 
one and a 'chaotic' one. The model Hamiltonian is diagonal in the regular subspace with equally spaced eigenvalues (representing Rydberg series). In the chaotic subspace (representing the perturbers), the Hamiltonian is modelled by a random matrix, with a coupling $v$ between regular and chaotic states ( $v$ in units of the spacing between regular states; for details see Ref. [10]). For large matrices, this model has only two parameters: the weight $\rho$ of chaotic states $(1-\rho$ of regular states) and the coupling strength $v$. Above the first ionization threshold, an imaginary part is added to the GOE matrix as in [10], with an additional parameter measuring the strength of coupling to the continua; this coupling strength is small playing therefore only a minor role.

The calculated NNS distributions for 1D-helium were fitted with this model, which turned out as a good description. The fits reproduce the lack of large spacings, and result in $\rho \cong 0.29,0.33$, and 0.40 for $I_{9}, I_{13}$, and $I_{17}$, respectively. A second estimate for $\rho$ is based on the size of the cut-off value for the level spacings (see Fig. $2(\mathrm{~d}-\mathrm{f})$ ), which can be related to $\rho$. In the perturbative regime, when the coupling between chaotic and regular levels is not so strong as to modify their densities, two neighboring states cannot be further apart than two unperturbed regular states. The reason is that a perturber repels neighboring levels and in this way reduces the NNS between them. With $m=\rho /(1-\rho)$ being the average number of chaotic states per regular state, the largest possible spacing will be $(m+1)=1 /(1-\rho)$ times the mean level spacing. This procedure leads to $\rho \cong 0.25$, 0.33 , and 0.41 for $I_{9}, I_{13}$, and $I_{17}$, respectively.

A further rough estimate for $\rho$ not based on the model, but on the physics of the real system, is possible: the local density of regular states can be estimated assuming that a Rydberg series converging to $I_{N}$ sees an effective nuclear charge of $Z-1=1$. The density of chaotic states is the sum of densities of states of all series with higher $N$. As $N$ increases, the upper thresholds lie closer and closer leading to an increase in the fraction of chaotic states. In this way, we obtain $\rho \cong 0.23,0.35$, and 0.43 , respectively, for $I_{9}, I_{13}$, and $I_{17}$. We note that all three approaches provide rather similar results for $\rho$.

The increase of $\rho$ with $N$ alone, however, is not sufficient to explain the transition to an almost Wignerlike distribution for $I_{17}$ : the coupling strength between chaotic and regular states has to increase too. This is indeed the case, with the best fits resulting in $v \cong 0.38$, 0.73 , and 1.2 for $I_{9}, I_{13}$, and $I_{17}$, respectively. It clearly shows that the individual influence of each perturber gets more important when one approaches higher thresholds. This leads to a globally chaotic spectrum, where a distinction between regular levels and perturbers loses more and more its meaning.

In conclusion, we have found - on the basis of statistical analysis - clear evidence of a transition towards quantum chaos in the doubly excitated spectrum of he- lium below $I_{9}$, with support from the results of our $a b$ initio calculations for 3D and $1 \mathrm{D}$ helium. The effects of chaos correspond to a loss of the radial quantum number $N$, whereas $N-K$ remains approximately a good quantum number, and they are directly related to the instability of the $e Z e$ orbits in radial direction (i.e preserving collinearity) and their stability with respect to bending. The statistical study of $1 \mathrm{D}$ helium provides an estimate for the observation of a fully chaotic regime in 3 D helium (for $N \geq 17$ ). It may happen that this regime appears even at lower $N$ values if $N-K$ breaks down. One can hope that future experiments as well as numerical calculations for $3 \mathrm{D}$ helium in the region above $I_{9}$ will provide further insight into the chaotic regime of helium.

The authors appreciate experimental assistance by J.D. Bozek as well as the constructive criticism of one of the referees. B.G. and D.D. thank M. Kuś for useful discussions. The work in Berlin was supported by the BMBF, project No. 05-650-KEA, and the DFG, project Do-561/1-3, that at ALS by the DOE, Contract No. DE-AC03-76SF00098. CPU time on a Cray C98 was provided by IDRIS. Laboratoire Kastler Brossel de l'Université Pierre et Marie Curie et de l'Ecole Normale Supérieure is UMR 8552 du CNRS.

[1] M. C. Gutzwiller, Rev. Mod. Phys. 70, 589 (1998).

[2] G. Tanner, K. Richter, and J.-M. Rost, Rev. Mod. Phys. 72, 497 (2000).

[3] T. Ericson, Phys. Rev. Lett. 5, 430 (1960).

[4] D. R. Herrick and O. Sinanoğlu, Phys. Rev. A 11, 97 (1975); C. D. Lin, Phys. Rev. A 29, 1019 (1984).

[5] M. Domke et al., Phys. Rev. Lett. 66, 1306 (1991).

[6] B. Grémaud and D. Delande, Europhys. Lett. 40, 363 (1997).

[7] A. Bürgers et al., J. Phys. B. 28, 3163 (1995).

[8] M.C. Gutzwiller, Chaos in Classical and Quantum Mechanics (Springer, New York, 1990).

[9] O. Bohigas in Chaos and Quantum Physics, edited by M.J. Giannoni, A. Voros, and J. Zinn-Justin, Les Houches Summer School, Session LII (North-Holland, Amsterdam, 1991).

[10] J. Zakrzewski, K. Dupret, and D. Delande, Phys. Rev. Lett. 74, 522 (1995); K. Dupret, J. Zakrzewski, and D. Delande, Europhys. Lett. 31, 251 (1995).

[11] R. Püttner et al., J. Electron Spectrosc. Rel. Phenom. 101-103, 27 (1999).

[12] M. Domke et al., Phys. Rev. A 53, 1424 (1996).

[13] F. Haake, Quantum Signatures of Chaos (Springer, Berlin, 1991).

[14] B. Grémaud and P. Gaspard, J. Phys. B 31, 1671 (1998).

[15] R. Blümel and W.P. Reinhard, Direction in Chaos 4 (1991); R. Blümel, Phys. Rev. A 54, 5420 (1996). 